Received: 2019.09.05

Accepted: 2019.11.14 Available online: 2020.01.21

Published: 2020.02.12

\title{
Management of Infected Bone Defects of the Lower Extremities by Three-Stage Induced Membrane Technique
}

Authors' Contribution: Study Design A Data Collection B Statistical Analysis C Data Interpretation D Manuscript Preparation E Literature Search F Funds Collection G

\author{
ABCDEF Chong Zhang \\ BCE Chunquan Zhu \\ F Guorong Yu \\ F Kai Deng \\ ACDEFG Li Yu
}

Department of Orthopedics Trauma and Microsurgery, Zhongnan Hospital of Wuhan University, Wuhan, Hubei, P.R. China
Corresponding Author: Source of support:

Background: Infected bone defects are therapeutic challenges. Although the induced membrane technique has been used for this problem, there is a $3 \%$ to $20.7 \%$ failure to eradicate infection, and there have been few reports about its use in tuberculous infection. We present our three-stage induced membrane technique (TSIMT) for treating infected bone defects of the lower extremity.

Material/Methods: Forty-one adult patients with infected bone defects of the lower extremities treated by TSIMT were included in a retrospective case-series study between January 2013 and June 2018. The clinical, imaging and laboratory assessment outcomes were analyzed.

Results: In the first stage, 3 patients had ankle tuberculous bone defects and 17 patients underwent 2-4 debridements. In the second stage, the average bone defect was $6.0 \mathrm{~cm}$; 1 patient needed an anterolateral thigh flap to cover the wound. In the third stage, 10 patients underwent autograft mixed allograft, and 18 cases used internal fixation. The mean follow-up period was 23.3 months. All patients achieved bone union and clinical eradication of infection. Changes in Lower Extremity Functional Scale (LEFS) scores after 1 year of TSIMT and bone union time are associated with advanced age, longer duration of infected bone defects, active smoking, and external fixation $(p<0.05)$, but are not dependent on bone defect size, debridement times, type of bone graft, or spacer-placing time ( $p>0.05)$.

Conclusions: TSIMT is effective in treating infected bone defects of the lower extremities. Advanced age, longer duration of infected bone defects, active smoking, and external fixation adversely affect bone union and recovery of infected extremities in a limited time span.

MeSH Keywords: Bone Diseases, Infectious • Polymethyl Methacrylate • Tuberculosis, Osteoarticular

Full-text PDF: https://www.medscimonit.com/abstract/index/idArt/919925

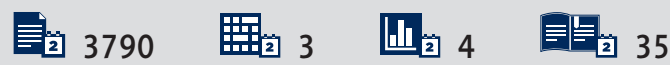




\section{Background}

Treatment of infected bone defects is challenging for orthopedic surgeons. Aggressive surgical debridement, appropriate antibiotic treatment, and reconstructive surgery play important roles in treatment of infected bone defects [1]. Radical debridement can reduce the failure rate and recurrence rate, but also inevitably creates segmental bone defects that are difficult to repair. Although distraction osteogenesis with external fixation, vascularized bone grafts, conventional bone grafting, and permanent acrylic spacers have been widely used, these techniques to reconstruct segmental bone defects are commonly associated with lengthy healing periods, complicated procedures, poor tolerance, and unpredictable complications. The induced membrane technique has excellent outcomes in treating significant bone defects and consists of 2 stages: 1) use of a polymethyl methacrylate (PMMA) spacer to fill the dead space to induce formation of a bioactive membrane, and 2) removal of the spacer and bone grafting [2]. Recently, Wu et al. [3] treated chronic osteomyelitis with two-stage management of the induced membrane technique, and promising results were reported. This two-stage management can also serve as an alternative for the treatment of infected bone defects [4].

However, the infected and nonviable tissues are sometimes difficult to differentiate from healthy tissue in a single debridement, and if the polymethylmethacrylate (PMMA) spacer is inserted at the same time, it can lead to recurrence of infection and failure of the induced membrane technique, as failure to eradicate infection has been reported at rates of 3-20.7\% $[3,5]$. Moreover, it is unnecessary to aggressively eliminate vascularized sclerotic tissue because the poorly vascularized parts can increase stability and decrease bone graft volume. Finally, collecting various tissue samples for bacterial culture and histopathological inspection, especially for some special pathogens (e.g., Mycobacterium tuberculosis), can aid diagnosis and treatment. Therefore, we use the three-stage induced membrane technique (TSIMT), including radical debridement and continuous irrigation with VSD, the insertion of a sensitive antibiotic-impregnated PMMA spacer, and the repair of bone defects.

We hypothesized that a functional outcome and thorough eradication of infection could be achieved by treating infected bone defects of the lower extremities with TSIMT. We aimed to assess the efficacy of TSIMT and identify factors that influence the outcomes of treatment.

\section{Material and Methods}

\section{Patients and inclusion criteria}

The Ethics Committee of our institution approved this study. The inclusion criteria were: (1) adult patients who were
Table 1. General patient data.

\begin{tabular}{|c|c|}
\hline Patient number (Male, Female), n & $41.0(31,10)$ \\
\hline Mean age (range), y & $47.3(29-66)$ \\
\hline Infected site (femur, tibia, ankle), n & $8,24,9$ \\
\hline $\begin{array}{l}\text { Average duration of infected bone } \\
\text { defects (range), } m\end{array}$ & $14.0(1-36)$ \\
\hline $\begin{array}{l}\text { Mean previous surgeries before the first } \\
\text { stage (range), } n\end{array}$ & $2.1(0-5)$ \\
\hline Mean final bone defect $(\mathrm{cm})$ & $6.0(3.0-10.0)$ \\
\hline Debridement times, $\mathrm{n}$ & $1.5(1-4)$ \\
\hline Duration of systemic antibiotics (week)* & $8.5(8-11)$ \\
\hline PMMA spacer placing time (weeks) & $13.6(10-24)$ \\
\hline Follow-up time (months) & $23.3(12-38)$ \\
\hline Mean initial mean LEFS score & $43.3(38-52)$ \\
\hline $\begin{array}{l}\text { Mean LEFS score at one year after stage } \\
\text { three }\end{array}$ & $58.9(49-69)$ \\
\hline Mean final mean LEFS score & $68.4(59-76)$ \\
\hline Union time (months) & $7.5(4-15)$ \\
\hline
\end{tabular}

* 3 patients with tuberculosis excluded.

diagnosed with infected bone defects of the lower extremities, (2) use of TSIMT as the treatment, and (3) minimum follow-up time of at least 1 year. The exclusion criteria were: (1) patients with diseases that can influence the healing of bone and functional reconstruction of the infected lower extremity, and (2) incomplete follow-up data. All data were retrospectively collected from the information system of our hospital, including medical history (such as smoking status), radiological data, and laboratory assessments.

From January 2013 to June 2018, 41 patients were included (31 men and 10 women, average age 47.3 years, age range 29-66 years). Twenty-five patients had infected bone defects of the lower extremities caused by surgery of open reduction of internal fixation, 13 patients had defects caused by open injury, and the remaining patients had defects caused by hematogenous infection. Twenty-four male patients were active smokers. The average duration of infected bone defects was 14.0 months (range 1-36 months). Before the first stage, a mean of 2.1 procedures (range $0-5$ ) had been performed for each patient, and all patients had received antibiotic treatment. There were 8,24 , and 9 cases of infections of the femur, tibia, and ankle, respectively. The infection sites of the femur and tibia were located on the diaphyses. Infected bone defects involving the distal tibia, astragalus, or/and calcaneus were defined as ankle infected bone defects (Table 1). 


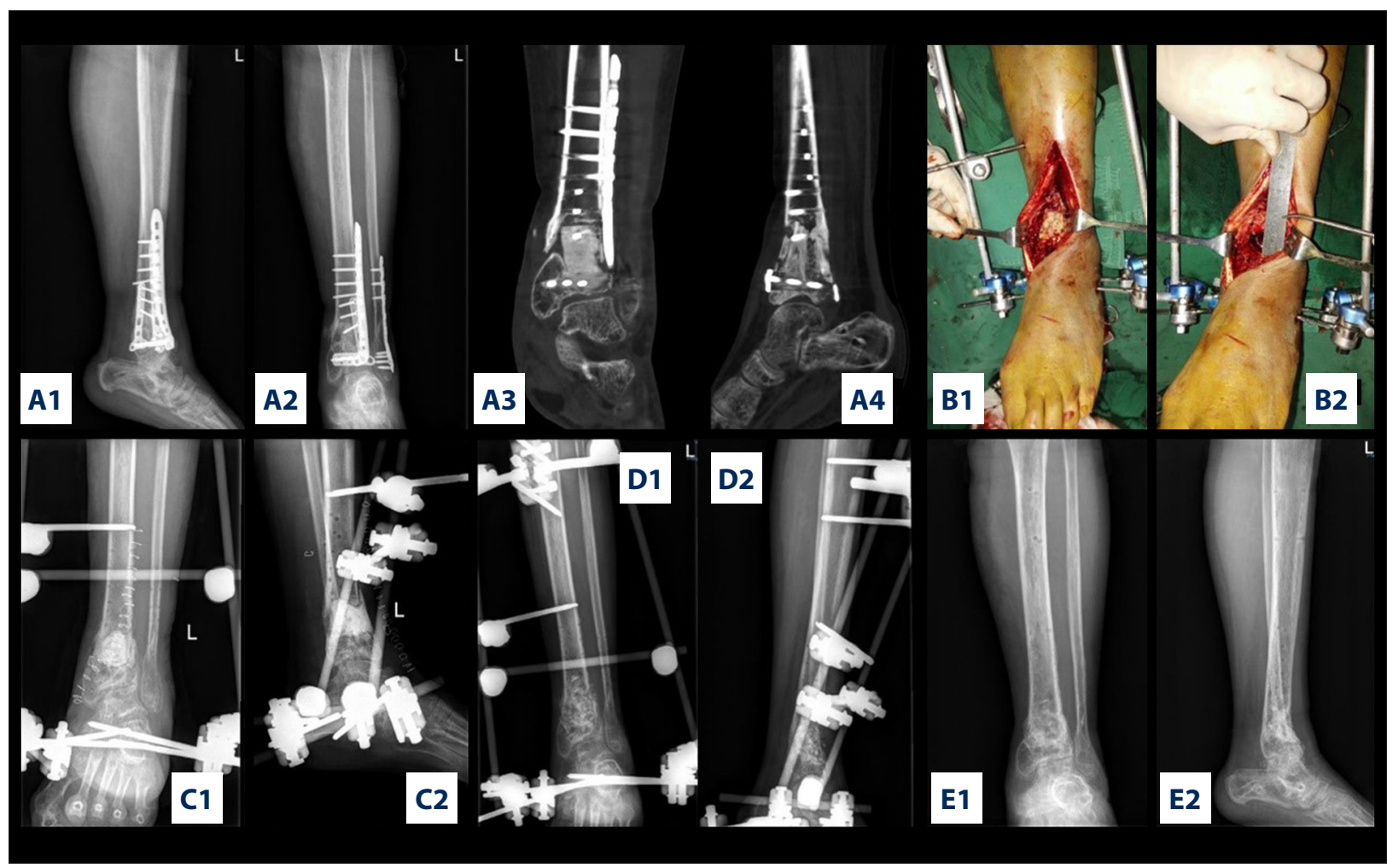

Figure 1. A representative case. A 54-year-old woman with tibial infected bone defect underwent TSIMT. Anteroposterior (A1, A3) and lateral (A2, A4) radiograph by X-ray and CT before first-stage surgery; (B1, B2) photos taken before and after debridement during the operation. Anteroposterior (C1, D1) and lateral $(\mathbf{C} 2, \mathbf{D} 2)$ radiographs after second- and third-stage surgery, respectively. Anteroposterior (E1) and lateral (E2) radiographs taken 9 months after third-stage surgery.

\section{Surgical technique}

\section{First-stage treatment protocol}

Following comprehensive clinical, biochemical, and radiological evaluation, all patients underwent TSIMT. Empiric broadspectrum antibiotic treatment were started when culture samples were sent for analysis. When the culture results were received, the antibiotics were changed to ones to which identified pathogens were sensitive.

The first-stage treatment consisted of multiple consecutive operative steps: radical debridement of infected tissues, lavage drainage with VSD, removal of the internal fixation material, and refixation with an external fixator, which depended on the stability of the bone.

The scope of radical debridement depended on preoperative and intraoperative investigations, such as imaging appearance, laboratory assessment (e.g., ESR and CRP), and clinical signs of infection (e.g., persistent pain and necrotic tissue of the infected site). In general, the margins of the debridement had to be beyond $5 \mathrm{~mm}$ of healthy bone and $2 \mathrm{~mm}$ of healthy soft tissue [6]. Different tissue samples were taken for bacterial culture and histopathological inspection at every debridement. External fixation was appropriately performed after debridement (Figures 1,2), except for patients who could have a stable structure without fixation (Figure 3). All patients underwent lavage drainage with VSD treatment for 1 week after debridement (more than $3000 \mathrm{ml}$ per day).

The number of radical debridements also depended on the degree of resolution of infection, including preoperative laboratory assessment (e.g., ESR and CRP) and culture results of lavage drainage and intraoperative investigations of surgery (e.g., persistence of necrotic tissue of the infected site). If the infection had been well-control, the patient received the treatment of the second stage; if not, the patient underwent repeat debridement surgery.

\section{Second-stage treatment protocol}

The second stage was the filling of dead space with antibiotic-impregnated PMMA spacers and reconstruction of softtissue defects. The choice of antibiotics was made according to the results of bacterial culture and histopathological inspection in stage one. Patients with general bacterial infection received $40 \mathrm{~g}$ PMMA with addition of $0.5 \mathrm{~g}$ gentamicin mixed 


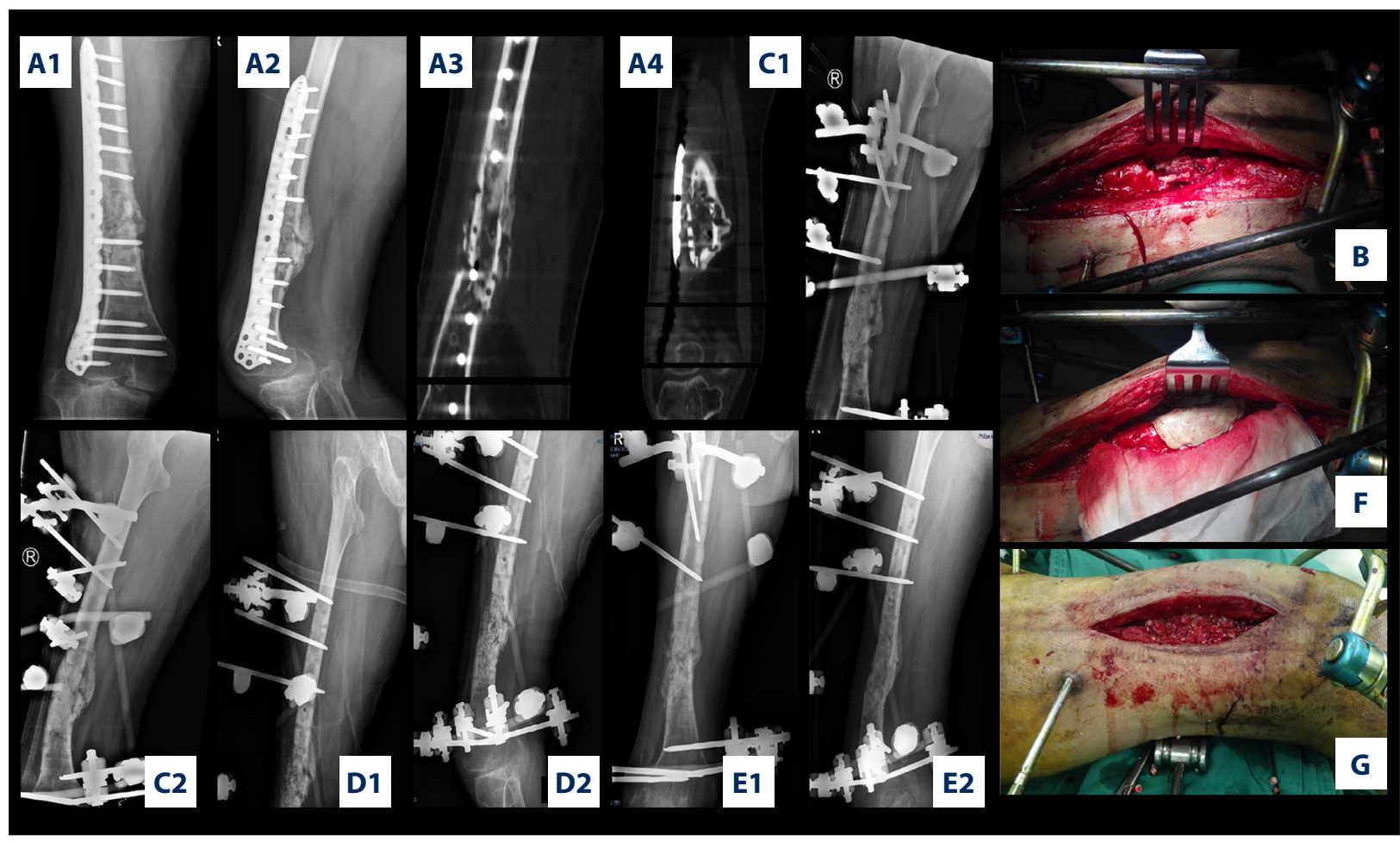

Figure 2. A representative case. A 62-year-old man with femoral infected bone defect underwent TSIMT. Anteroposterior (A1, A3) and lateral (A2, A4) radiograph by X-ray and CT before first-stage surgery; anteroposterior (C1, D1) and lateral (C2, D2) radiographs after second- and third-stage surgery, respectively. (B, F, G) Photos taken after debridement, insertion of PMMA, and bone graft. Anteroposterior (E1) and lateral (E2) radiographs taken 10 months after third-stage surgery.

with 2-3 g Vancomycin powder, and those with tuberculous infection also received $1 \mathrm{~g}$ streptomycin powder. Patients received intravenous antibiotic therapy throughout the first 2 stages. Most patients were discharged after 2 weeks of the second-stage treatment and usually received at least 4 weeks of oral antibiotic therapy, except for patients with tuberculosis infection, who underwent least 1 year of antituberculosis therapy. Muscular or fasciocutaneous flaps (e.g., anterolateral thigh flap) were used for patients with soft-tissue defects (Figure 4). Subsequently, patients performed partial weightbearing rehabilitation to avoid osteoporosis.

\section{Third-stage treatment protocol}

When normal values of ESR and CRP, and healing of the sinus tract and flap were observed, the third stage was performed, which consisted of removal of the spacer, morselized bone grafting with an autograft from the ilium/fibula or a mixed allograft, and change of final fixation.

When the status of surrounding soft tissue was good and the discomfort level was acceptable, patients received an internal fixator (e.g., LCP plate) as the final fixation. If the articular surface had been destroyed, articular fusion using internal fixation was performed (e.g., ankle infected bone defect). Cancellous bone autograft from an iliac crest and fibula (Figure 3) was usually the best choice. If insufficient material was available, combining a cancellous bone autograft with allograft (less than $40 \%)$ was also an alternative.

\section{Postoperative follow-up}

Postoperative radiologic images and clinical results of treatment, including the rate of cure, time to union, the Lower Extremity Functional Scale (LEFS) [7] were recorded. Bone union was defined by 2 continuous cortices on 2 different views. Infection cure was defined by eradication of the clinical infectious signs and normalization of ESR and CRP. The LEFS consisted of 20 functional leg activities, of which the highest score was 80 .

\section{Statistical analysis}

Quantitative and qualitative variables were reported separately as mean and standard deviation and number. Data were analyzed using the SPSS 21.0 statistical software (SPSS, Inc., Chicago, IL, USA). An independent-samples $t$ test was used for comparison of continuous variables. The significance threshold was set at 0.05 in a two-sided test (Table 2). 


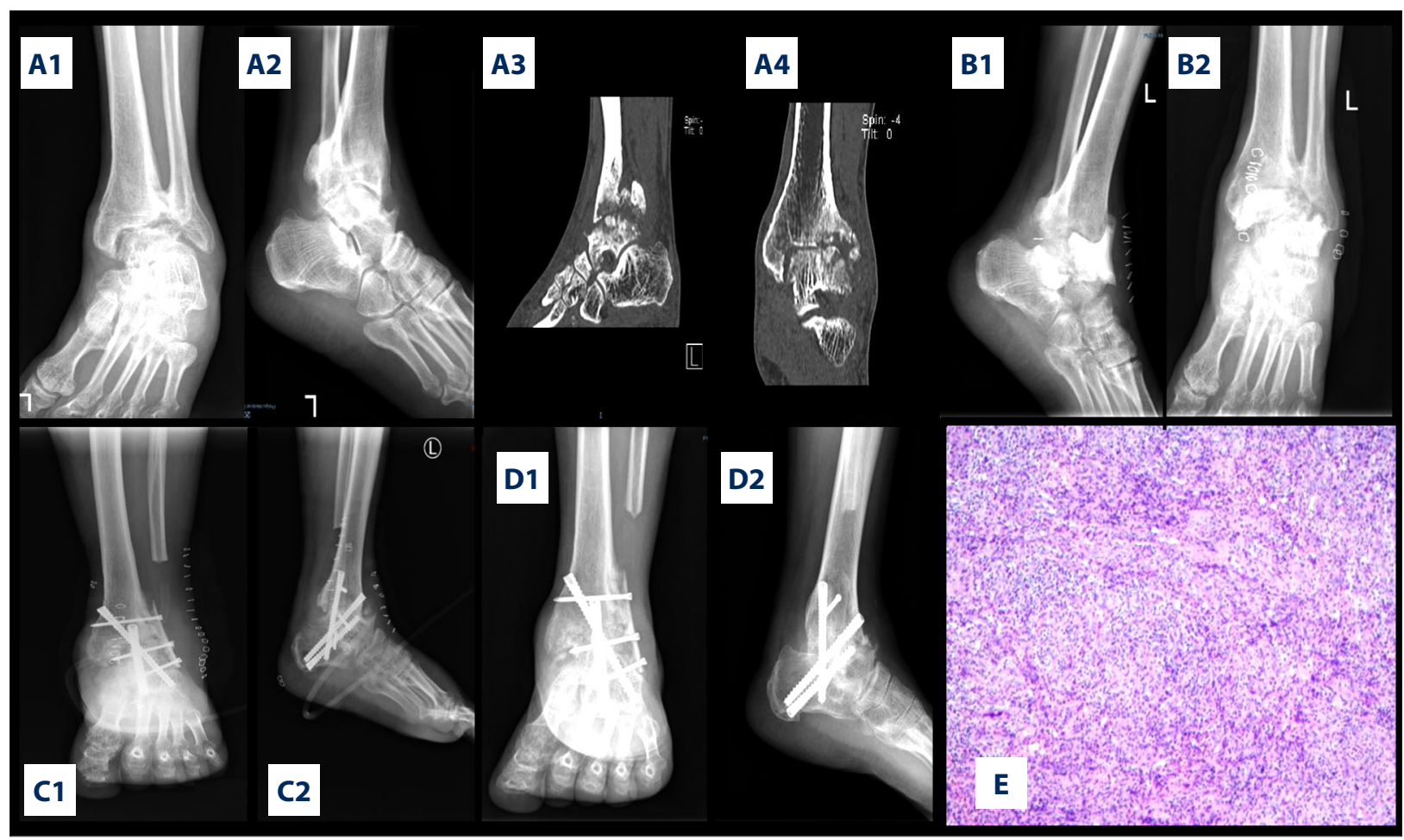

Figure 3. A representative case. A 50-year-old man with ankle infected bone defect caused by tubercle bacillus underwent TSIMT. Anteroposterior (A1, A3) and lateral (A2, A4) radiographs by X-ray and CT before first-stage surgery; anteroposterior (B1, C1) and lateral $(B 2$, C2) radiograph after second- and third-stage surgery, respectively. Anteroposterior (D1) and lateral (D2) radiographs taken 10 months after third-stage surgery. (E) Pathologic findings.

\section{Results}

In the first stage, 17 patients underwent debridement 2-4 times. One patient with a femur infected bone defect underwent 4 debridements, and 2 debridements were performed for 3 patients with femur defects, 10 patients with tibia infected bone defect, and 3 patients with ankle infected bone defect. Only 3 patients with ankle infected bone defects; they did not receive fixation because the bone defects could be treated as lacunar bone defects and the bone construction can remain stable after debridement, which reached the demand for treatment, so they did not need to get external fixation. The remaining patients all received an external fixator for stabilizations. After the first stage, 34 patients had positive cultures; one of them and 2 patients with negative culture results were diagnosed with Mycobacterium tuberculosis infection in the ankle according to the biopsy results (Table 3 ).

The second stage was performed after a mean of 1.5 weeks (range 1-4 weeks). In the second stage, all results of bacterial culture were negative. The final bone defects after debridement had a mean size of $6.0 \mathrm{~cm}$ (range $3.0-10.0 \mathrm{~cm}$ ). According to the results of the bacterial culture and histopathological inspection of the first stage, vancomycin powder was mixed with PMMA for 38 patients, and 3 patients received vancomycin and streptomycin powder. Only 1 patient with an ankle infected bone defect had an anterolateral thigh flap placed to cover the soft-tissue defect. The mean spacer placing time was 13.6 weeks (range 10-24 weeks).

At the third stage, 4 patients with femur infected bone defect and 6 patients with tibial infected bone defect received ilium and allograft as bone grafts, 9 patients with ankle infected bone defect received fibula as bone grafts, and the remaining patients all received bone autografts from iliac crests. For final fixation, 5 patients with femur infected bone defect and 4 patients with tibial infected bone defect received internal fixation (LCP plate) as the final fixation. Articular fusion using internal fixation was performed in the 9 patients with ankle infected bone defect, such as tibio-talo-calcaneal fusion. There were no intra- or postoperative complications.

Forty-one patients underwent a mean of 8.5 weeks (range 8-11 weeks) of antibiotic therapy in total, and 3 patients with tuberculosis underwent least 1 year of antituberculosis therapy. The mean follow-up period was 23.3 months (range 12-38 months). Bony union was achieved in $100 \%$ of cases, with a mean of 7.5 months (range 4-15 months after the third stage). No recurrence was found, and no more supplementary procedures were required. The mean LEFS scores before the first 


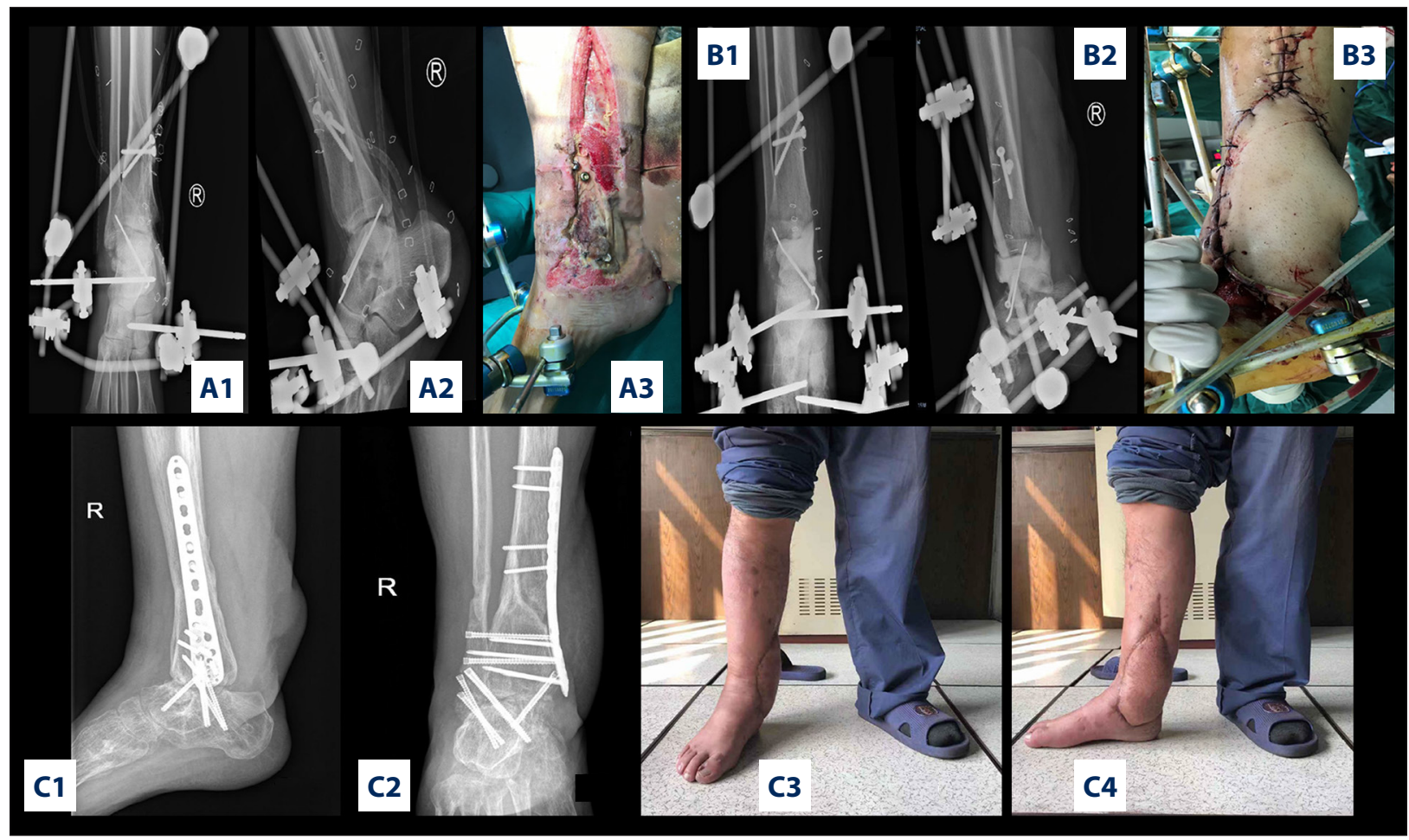

Figure 4. A representative case. A 38-year-old man with ankle acute infected bone defect and a significant soft-tissue defect underwent TSIMT. Anteroposterior (A1) and lateral (A2) radiographs by X-ray before the first stage; (A3) was taken at the first debridement surgery; anteroposterior (B1) and lateral (B2) radiographs after the second stage; (B3) was taken after the second stage with the flap. Anteroposterior $(\mathbf{C} 1, \mathbf{C} 3)$ and lateral $(\mathbf{C 2}, \mathbf{C} 4)$ radiographs taken 10 months after third-stage surgery.

stage, at 1 year after stage 3 and at the last follow-up were 43.3 (38-52), 58.9 (49-69), and 68.4 (59-76), with an average of $85.5 \%$ (range $73.8-95 \%$ ) of maximal function (Table 1).

By analysis of the related factors that can affect the bone union time and the change of LEFS after 1 year of the third stage, we discovered that bone defect size, type of bone graft, debridement times, and spacer-placing time did not influence bone union time or the change of LEFS after 1 year of the third stage ( $p>0.05)$. Advanced age, longer duration of infected bone defects, active smoking, and external fixation method had an adverse impact on bone union time and the change of LEFS after 1 year of the third stage $(p<0.05)$ (Table 2$)$.

\section{Discussion}

\section{Principle of treatment and reasons why we use TSIMT for infected bone defects of the lower extremities}

With rising numbers of surgeries and use of implants worldwide, the number of infections is rising [8]. Infected bone defects are characterized by a progressive inflammatory process caused by pathogens, leading to bone destruction and sequestrum formation. Difficulties in treatment concern 2 aspects: 1) control of the infection, and 2) addressing osseous defects [9]. Aggressive surgical debridement, appropriate antibiotic treatment, and reconstructive surgery play important roles in dealing with infected bone defects and in ensuring longterm success [1]. Concerning debridement, more surgeons are aware of the importance of radical debridement to remove all devitalized and infected tissues, including skin, sinus tract, soft tissue envelope, and bone, until vital bleeding tissue remains ('paprika sign') [10]. Radical debridement should not be limited by the fear of enlarging the tissue defects, as inadequate debridement has a high rate of recurrence $[11,12]$.

Although the induced membrane technique has been widely used for infected bone defects, the reasons why we used TSIMT were as follows: 1) As infected, nonviable tissue may be hard to differentiate from normal tissue by one debridement firstly, serial debridement with VSD to eradicate the nidus is necessary to prevent a recurrence [13]. 2) To eradicate the infection completely, using the two-stage treatment, the scope of debridement may far exceed the actual debridement range. All necrotic bone was removed down to bleeding bone ('paprika sign'), but sclerotic bone need not necessarily be sacrificed as long as it is vascularized. Even poorly vascularized parts can 
Table 2. Outcome between different groups*.

\begin{tabular}{|c|c|c|c|c|}
\hline Group (n) & $\begin{array}{l}\text { Bone union time } \\
\text { (months) }\end{array}$ & P value & $\begin{array}{l}\text { Difference of } \\
\text { LEFS** }\end{array}$ & $P$ value \\
\hline \multicolumn{5}{|l|}{ Age } \\
\hline$<40$ y $(15)$ & $5.60 \pm 0.99$ & \multirow{2}{*}{0.000} & $18.6 \pm 4.10$ & \multirow{2}{*}{0.001} \\
\hline$\geq 40$ y $(26)$ & $8.65 \pm 2.92$ & & $13.8 \pm 3.75$ & \\
\hline \multicolumn{5}{|c|}{ Duration of infected bone defects } \\
\hline$<12$ months (13) & $5.31 \pm 1.03$ & \multirow{2}{*}{0.000} & $18.2 \pm 5.12$ & \multirow{2}{*}{0.009} \\
\hline$\geq 12$ months (28) & $8.57 \pm 2.78$ & & $14.4 \pm 3.61$ & \\
\hline \multicolumn{5}{|l|}{ Smoking } \\
\hline Yes (24) & $8.54 \pm 3.04$ & \multirow{2}{*}{0.002} & $13.7 \pm 3.51$ & \multirow{2}{*}{0.001} \\
\hline No (17) & $6.12 \pm 1.69$ & & $18.3 \pm 4.37$ & \\
\hline \multicolumn{5}{|l|}{ Debridement times } \\
\hline 1 time (24) & $7.25 \pm 2.75$ & \multirow{2}{*}{0.445} & $16.1 \pm 3.82$ & \multirow{2}{*}{0.427} \\
\hline$\geq 2$ times (17) & $7.94 \pm 2.93$ & & $14.9 \pm 5.30$ & \\
\hline \multicolumn{5}{|l|}{ Length of bone defect } \\
\hline$<5 \mathrm{~cm}(8)$ & $6.88 \pm 3.05$ & \multirow{2}{*}{0.465} & $15.8 \pm 6.94$ & \multirow{2}{*}{0.947} \\
\hline$\geq 5 \mathrm{~cm}(33)$ & $7.70 \pm 2.78$ & & $15.6 \pm 3.79$ & \\
\hline \multicolumn{5}{|l|}{ Type of bone graft } \\
\hline Autograft (31) & $7.26 \pm 2.63$ & \multirow{2}{*}{0.269} & $16.3 \pm 4.01$ & \multirow{2}{*}{0.071} \\
\hline Mixed bone graft $(10)^{\#}$ & $8.40 \pm 3.31$ & & $13.4 \pm 5.27$ & \\
\hline \multicolumn{5}{|l|}{ Final stabilization method } \\
\hline Internal fixation (18) & $6.06 \pm 1.66$ & \multirow{2}{*}{0.001} & $18.0 \pm 4.26$ & \multirow{2}{*}{0.002} \\
\hline External fixation (23) & $8.70 \pm 3.01$ & & $13.7 \pm 3.73$ & \\
\hline \multicolumn{5}{|l|}{ Spacer placing time } \\
\hline$<12$ weeks (9) & $7.33 \pm 2.12$ & \multirow{2}{*}{0.810} & $15.0 \pm 4.24$ & \multirow{2}{*}{0.649} \\
\hline$\geq 12$ weeks (32) & $7.59 \pm 3.00$ & & $15.7 \pm 4.58$ & \\
\hline
\end{tabular}

* Analyzed using an independent-samples $t$ test; ${ }^{* *}$ difference of LEFS=LEFS measured about one year after stage 3 -LEFS measured at the beginning of stage one; \# autograft and allograft.

serve an important role in the stability and decrease the volume of bone grafts. 3) using three-stage treatment, we had the time to take different tissue samples for bacterial culture and histopathological inspection, providing the best possibility to use a sensitive antibiotic systemically and locally to decrease recurrence, especially for some special pathogens such as Mycobacterium tuberculosis. At the same time, we could reassess the condition several times and decide whether it was appropriate to proceed to the next stage, decreasing the rate of recurrence.

\section{How to use antibiotics systematically and locally?}

Antibiotic therapy exerts its full adjunctive effect after radical surgical debridement as a treatment for residual pathogens. Empiric antibiotic treatment must start as soon as the culture samples have been obtained if there is a sinus or soft-tissue defects. In the case of culture-negative infection, a second-generation cephalosporin is recommended until the results of intraoperative bacterial culture and histopathological inspection are obtained. Most authors recommend 4-6 weeks of antibiotic therapy, which is based on the time (3-4 weeks) needed 
Table 3. Bacteria isolated and pathological report in stage 1.

\begin{tabular}{|c|c|c|}
\hline Number of patients & Bacteria & Pathological report \\
\hline 9 & S. aureus & $\begin{array}{l}\text { Chronic osteomyelitis ( } 8 \text { patients), chronic } \\
\text { osteomyelitis+Granulomatous inflammation (1 patient) }\end{array}$ \\
\hline 4 & MRSA & Chronic osteomyelitis \\
\hline 4 & S. epidermidis & Chronic osteomyelitis \\
\hline 3 & E. cloacae & Chronic osteomyelitis \\
\hline 4 & P. aeruginosa & Chronic osteomyelitis \\
\hline 1 & A. baumannii & Chronic osteomyelitis \\
\hline 2 & K. pneumoniae & Chronic osteomyelitis \\
\hline 2 & P. mirabilis & Chronic osteomyelitis \\
\hline 2 & S. aureus, E. cloacae & Chronic osteomyelitis \\
\hline $1^{*}$ & S. aureus, E. faecalis & Chronic osteomyelitis \\
\hline $1^{*}$ & C. sporogenes, $P$. aeruginosa & Chronic osteomyelitis \\
\hline 1 & S. aureus, $P$. aeruginosa, E. cloacae & Chronic osteomyelitis \\
\hline 7 & Negative culture result & $\begin{array}{l}\text { Chronic osteomyelitis ( } 5 \text { patients), } \\
\text { osteomyelitis+Granulomatous inflammation ( } 2 \text { patients) }\end{array}$ \\
\hline
\end{tabular}

MSSA - methicillin-susceptible Staphylococcus aureus. * The bacteria isolated from first and second debridement is S. aureus/C. sporogenes and E. cloacae/P. aeruginosa.

for the revascularization of the bone, which causes the antibiotics to work more effectively [14]. All recommendations are empirical, and the duration of concomitant systemic antibiosis and the positive effects of prolonged antibiotic therapy are still unclear [14]. In fact, prolonged antibiotic treatment increases the risk of adverse effects and antibiotic resistance, even for planktonic bacteria [14]. Planktonic bacteria may be eliminated rather quickly; however, biofilm-embedded pathogens require up to 1000 times the minimum inhibitory concentration (MIC), and it is usually not possible to achieve this high of a therapeutic concentration with systemic antibiotic therapy [15].

To reduce systemic adverse effects and provide an effective local concentration of antibiotics for the treatment of infected bone defects [16], the combination of systemic and local antibiotic treatment becomes preferable and may be a valuable tool against orthopedic infections. Research has also found that antibiotic administration through the oral or parenteral route makes no difference when treating infected bone defects if the bacteria are susceptible to the antibiotic used [11].

A local carrier consisting of antibiotics and PMMA provides a much higher concentration of antibiotics to the surrounding tissues than does intravenous antibiotic therapy [17,18]. With regard to the choice of antibiotics to mix with PMMA, the results of bacterial culture and histopathological inspection matter greatly. Glycopeptides and aminoglycosides are most commonly used [9] because they are broad-spectrum, high local concentrations are released from PMMA cement, and they are thermostable [1]. Gram-positive pathogens that cause bone infection are mainly susceptible to vancomycin. Most gram-negative bacteria are susceptible to tobramycin [9]. Vancomycin and tobramycin have the lowest cytotoxic effect [19] and rare systemic adverse effects after local application [20]. Moreover, the type and extent of infected bone defects, medical comorbidities, isolated organism(s), and whether the infection is recurrent are very important [21]. In polymicrobial infections, a combination of antibiotics can be used to reduce the recurrence rate [22]. Even for patients with strong evidence of monomicrobial infection (symptoms with the typical clinical appearance and unambiguous culture), it seems best to reserve application of a single local antibiotic in order to address the problem of potentially undetected polymicrobial infection [9]. Four grams of vancomycin per $40 \mathrm{~g}$ of PMMA cement was used for the infected bone defects [23]. An average of 3.4 batches of cement with a mean of $10.5 \mathrm{~g}$ of vancomycin and $12.5 \mathrm{~g}$ of gentamicin has been used for infected bone defects without systemic toxicity [16]. Therefore, the $2 \mathrm{~g}$ of vancomycin were mixed with per $40 \mathrm{~g}$ of PMMA with the addition of $0.5 \mathrm{~g}$ of gentamicin for general infection, and $2 \mathrm{~g}$ of vancomycin and $1 \mathrm{~g}$ of streptomycin with $40 \mathrm{~g}$ of PMMA with addition of $0.5 \mathrm{~g}$ of gentamicin for patients with tuberculosis were used. 


\section{Advantages and best timing of PMMA spacer}

Apart from providing extremely high concentrations of antibiotics, PMMA can fill the dead space and induce the formation of a membrane [17]. The induced membrane technique is simple and useful for addressing critical bone size defects, with a high rate bone union that ranges from $82.6 \%$ to $100 \%[2,24]$. One-stage cancellous bone grafts can undergo bone necrosis and resorption when the bone defects exceeds $2 \mathrm{~cm} \mathrm{[25].}$ However, the induced membrane technique can revascularize the bone graft and prevent bone resorption; moreover, the induced membrane is highly vascularized, which can provide an osteogenic microenvironment to support bone lateral regeneration by secreting several growth factors, including VEGF and BMP-2 [2]. Research also found that the cement spacer can prevent fibrous tissue from invading into the bone defect site, provide stability, and facilitate the repairing of the affected soft tissue, which is helpful for the eradication of infection [26]. Complete soft-tissue coverage is critical for the formation of the membrane, and muscular or fasciocutaneous flaps can be used if necessary. Muscle flaps are preferable for reducing the recurrence rates of chronic infected bone defects because they not only can manage dead space and provide soft-tissue coverage, but also prevent contamination, improve vascularity, and enhance the delivery of antibiotics [27].

There is no consensus regarding the timing of PMMA spacer removal and bone grafting [24]. Currently, 4 to 8 weeks is thought to be the best time for bone graft because this period has the highest level of vascularization and growth factors and the best status of the induced membrane [2]. Auregan et al. [26] found that the best timing to approach the induced membrane depended on the vascularization of the segment; when more vascular axes vascularized the segment, the membrane was vascularized and usable earlier. Therefore, the time interval is longer in femurs and ankles compared with tibias. Due to the inflammation (ESR and CRP), poor soft-tissue coverage, and type of pathogens, the timing of PMMA spacer removal in our study was later, at least 10 weeks, and approximately 6 months in patients with tubercle bacillus.

\section{Bone grafts and stabilization}

For bone grafts, cancellous bone autografts from an iliac crest and fibula are the best choice. If not enough material is available, combining a cancellous bone autograft with allograft (less than 40\%) is also an alternative [26]. Cancellous bone can also be obtained through the technique of RIA (reaming irrigation aspiration) [28].

The option of bone stabilization is influenced by several factors, including the characteristics of the defects (e.g., the severity of infection and the location and size), the status of the affected soft tissue, firm fixation needed for therapy, and the patient's tolerance [3]. Along with debridement, the infected or unhealthy tissues should be considered to be contaminated, and the surface of internal implants can act as a substratum for the attachment of bacteria and formation of biofilms [29]. Therefore, in the first stage, traditional external fixation was treated as the best choice for eradication of the infection and protection of the affected soft tissue. Studies have also used locking compression plates as external fixation, which are versatile and have good patient tolerance and satisfactory outcomes [30,31]. Additionally, bone resorption and nonhealing have been reported to occur in cases with low stable fixation [32]. As long as the local antibiotic concentrations are higher than the dosage required for eliminating biofilmembedded bacteria (more than $100 \mathrm{mg} / \mathrm{l}$ ), the risk of contamination of alloplastic material is low [9]. When mixing bone grafts with antibiotics, their storage capability for antibiotics exceeds those of PMMA and other carriers, and the entire amount is available, leading to high local concentrations and a prolonged biofilm-active release [9]. These capacities make reconstruction and internal stabilization at the same time possible in the third stage.

\section{What factors influence the outcomes of treatment?}

We analyzed the related factors involved in the bone union time and improvement of function of the lower extremities, which was defined as the difference between LEFS measured before first stage and about 1 year after the third stage, and discovered that defect size, type of bone graft, debridement times, and spacer-placing time did not influence bone union time or the change in LEFS after 1 year of the third stage. The findings were previously reported $[3,33,34$. The bone union time and improvement of lower-extremities function in a limited time were more affected by age, duration of infected bone defects, active smoking, and final fixation method in our series. As with smoking, longer duration of infected bone defects means longer immobilization, persistence of infection, and more surgeries that can destroy local blood supply and have an adverse impact on bone union and improvement of function of the lower extremities. Research [35] found that smoking significantly increased the risk of nonunion of fractures but nonsignificantly increased time to union in all fractures, and the impact of smoking on bone union time found in our research may be attributable to our small sample size. Internal fixation can provide sufficient stabilization and allow resumed weightbearing owing to the good toleration, which can facilitate the union and recovery of function. Although research found that age did not influence lower-extremity improvement [3], the effect of the involved factors on the improvement of LEFS was not time-limited. With a long duration of rehabilitation, the impact of age on lower-extremity improvement may be less significant, but further research is needed. 
The present study has certain limitations. First, the study population in our research was small and it was a retrospective study. Second, some variables were not assessed, such as the joint range of motion. Randomized controlled studies with larger sample sizes are needed.

\section{Conclusions}

TSIMT showed excellent outcomes in the treatment of infected bone defects of the lower extremities, which can be used to radically clean the infected sites, decrease the rate of recurrence, and effectively facilitate the reconstruction of bone defects. Advanced age, longer duration of infected bone defects, active smoking, and external fixation method had an adverse effect on bone union time and the recovery of infected extremities in a limited time (1 year after TSIMT). Further research is needed to determine the best time for bone grafting, more appropriate skeletal fixation, and to assess long-term effects of the factors involved on recovery of the infected extremities.

\section{Conflict of interests}

None.

\section{References:}

1. Rao N, Ziran BH, Lipsky BA: Treating osteomyelitis: Antibiotics and surgery. Plast Reconstr Surg, 2011; 127(Suppl. 1): 177s-87s

2. Masquelet AC, Begue T: The concept of induced membrane for reconstruction of long bone defects. Orthop Clin North Am, 2010; 41(1): 27-37

3. Wu H, Shen J, Yu X et al: Two-stage management of Cierny-Mader type IV chronic osteomyelitis of the long bones. Injury, 2017; 48(2): 511-18

4. Han W, Shen J, Wu H et al: Induced membrane technique: Advances in the management of bone defects. Int J Surg, 2017; 42: 110-16

5. Raven TF, Moghaddam A, Ermisch C et al: Use of Masquelet technique in treatment of septic and atrophic fracture nonunion. Injury, 2019; 50(Suppl.) 340-54

6. Simpson AH, Deakin M, Latham JM: Chronic osteomyelitis. The effect of the extent of surgical resection on infection-free survival. J Bone Joint Surg Br, 2001; 83(3): 403-7

7. Mehta SP, Fulton A, Quach C et al: Measurement properties of the lower extremity functional scale: A systematic review. J Orthop Sports Phys Ther 2016; 46(3): 200-16

8. Hogan A, Heppert VG, Suda AJ: Osteomyelitis. Arch Orthop Trauma Surg, 2013; 133(9): 1183-96

9. Winkler H: Treatment of chronic orthopaedic infection. EFORT Open Rev, 2017; 2(5): 110-16

10. Fang RC, Galiano RD: Adjunctive therapies in the treatment of osteomyelitis. Semin Plast Surg, 2009; 23(2): 141-47

11. Conterno LO, Turchi MD: Antibiotics for treating chronic osteomyelitis in adults. Cochrane Database Syst Rev, 2013; (9): Cd004439

12. Mouzopoulos G, Kanakaris NK, Kontakis G et al: Management of bone infections in adults: The surgeon's and microbiologist's perspectives. Injury, 2011; 42(Suppl. 5): S18-23

13. Eckardt JJ, Wirganowicz PZ, Mar T: An aggressive surgical approach to the management of chronic osteomyelitis. Clin Orthop Relat Res, 1994; (298): 229-39

14. Bernard L, Dinh A, Ghout I et al: Antibiotic treatment for 6 weeks versus 12 weeks in patients with pyogenic vertebral osteomyelitis: An open-label, non-inferiority, randomised, controlled trial. Lancet, 2015; 385(9971): 875-82

15. Saginur R, Stdenis M, Ferris $W$ et al: Multiple combination bactericidal testing of staphylococcal biofilms from implant-associated infections. Antimicrob Agents Chemother, 2006; 50(1): 55-61

16. Springer BD, Lee GC, Osmon D et al: Systemic safety of high-dose antibiotic-loaded cement spacers after resection of an infected total knee arthroplasty. Clin Orthop Relat Res, 2004; (427): 47-51

17. Hake ME, Young H, Hak DJ et al: Local antibiotic therapy strategies in orthopaedic trauma: Practical tips and tricks and review of the literature. Injury, 2015; 46(8): 1447-56

18. Hanssen AD: Local antibiotic delivery vehicles in the treatment of musculoskeletal infection. Clin Orthop Relat Res, 2005; (437): 91-96

19. Edin ML, Miclau T, Lester GE et al: Effect of cefazolin and vancomycin on osteoblasts in vitro. Clin Orthop Relat Res, 1996; (333): 245-51

20. Buttaro MA, Gimenez MI, Greco $G$ et al: High active local levels of vancomycin without nephrotoxicity released from impacted bone allografts in 20 revision hip arthroplasties. Acta Orthop, 2005; 76(3): 336-40

21. Panteli M, Giannoudis PV: Chronic osteomyelitis: What the surgeon needs to know. EFORT Open Rev, 2016; 1(5): 128-35

22. Spellberg B, Lipsky BA: Systemic antibiotic therapy for chronic osteomyelitis in adults. Clin Infect Dis, 2012; 54(3): 393-407

23. Zalavras CG, Patzakis MJ, Holtom P: Local antibiotic therapy in the treatment of open fractures and osteomyelitis. Clin Orthop Relat Res, 2004; (427): 86-93

24. Taylor BC, Hancock J, Zitzke R, Castaneda J: Treatment of bone loss with the induced membrane technique: Techniques and outcomes. J Orthop Trauma 2015; 29(12): 554-57

25. Tiemann AH, Schmidt HG, Braunschweig R, Hofmann GO: Strategies for the analysis of osteitic bone defects at the diaphysis of long bones. Strategies Trauma Limb Reconstr, 2009; 4(1): 13-18

26. Auregan JC, Begue T: Induced membrane for treatment of critical sized bone defect: A review of experimental and clinical experiences. Int Orthop, 2014; 38(9): 1971-78

27. Patzakis MJ, Zalavras CG: Chronic posttraumatic osteomyelitis and infected nonunion of the tibia: Current management concepts. J Am Acad Orthop Surg, 2005; 13(6): 417-27

28. Giannoudis PV, Tzioupis C, Green J: Surgical techniques: How I do it? The Reamer/Irrigator/Aspirator (RIA) system. Injury, 2009; 40(11): 1231-36

29. Cierny $G 3^{\text {rd }}$, Mader JT, Penninck JJ: A clinical staging system for adult osteomyelitis. Clin Orthop Relat Res, 2003; (414): 7-24

30. Xiao C, Tang F, Zhou Y et al: A locking compression plate as an external fixator for treating infected nonunion of the humeral diaphysis. BMC Surg, 2016; 16(1): 53

31. Zhang J, Ebraheim NA, Li M et al: External fixation using a locking plate: A reliable way in treating distal tibial fractures. J Orthop Trauma, 2015; 29(11): e454-58

32. Accadbled F, Mazeau P, Chotel F et al: Induced-membrane femur reconstruction after resection of bone malignancies: Three cases of massive graft resorption in children. Orthop Traumatol Surg Res, 2013; 99(4): 479-83

33. Karger $C$, Kishi T, Schneider $L$ et al: Treatment of posttraumatic bone defects by the induced membrane technique. Orthop Traumatol Surg Res, 2012; 98(1): 97-102

34. Siboni R, Joseph E, Blasco L et al: Management of septic non-union of the tibia by the induced membrane technique. What factors could improve results? Orthop Traumatol Surg Res, 2018; 104(6): 911-15

35. Scolaro JA, Schenker ML, Yannascoli $S$ et al: Cigarette smoking increases complications following fracture: A systematic review. J Bone Joint Surg Am, 2014; 96(8): 674-81 